inmunostained, were assigned a relative value from 0 (minimalstaining)to 4 (most intense inmunostaining). Quantitative morphometric assessment was done on coded slides with $400 x$ magnification and a eye piece with a sample square grid pattern (model CPLW 1018, Zeiss Optical, Hannover Md) and was done following the mathematical model of Weibel. Differences between the groups were determined by one way ANOVA $(p<0.01)$.

Results: The VEGF was significantly decreased in the lungs of rats recovered in hyperoxia, it was correlated with a lower degree of alveolarization. InhaledNOtreatmentafterhyperoxianeitherincrease lung VEGF expression nor alveolarization.

Conclusion: The inhaled NO did not improve the changes observed in rat lungs after hyperoxia exposure.

490

\section{MATRIX METALLOPROTEINASE 2, TIMP- 1 AND TIMP-2 CONCENTRATIONS IN TRACHEAL ASPIRATE FLUID AND PLASMA OF PRETERM INFANTS DEVELOPING BRONCHOPULMONARY DYSPLASIA}

M. Gach ${ }^{1}$, M. Rybak-Krzyszkowska², W. Fendler ${ }^{3}$, R. Lauterbach ${ }^{4}$, A. Piotrowski ${ }^{1}$

${ }^{1}$ Pediatric Anesthesiology and Intensive Care Department, Medical University of Lodz, Lodz,

${ }^{2}$ Department of Obstetrics and Perinatology, Jagiellonian University Medical College, Krakow, ${ }^{3}$ Department of Immunopathology and Genetics, Medical University of Lodz, Lodz, ${ }^{4}$ Department of Neonatology, Jagiellonian University Medical College, Krakow, Poland

Background and aims: Bronchopulmonary Dysplasia (BPD) is one of the most important complications of prematurity. MMPs are a group of proteolytic enzymes involved in lung development. Imbalance between MMPs and their inhibitors has been implicated in increased inflammation and impaired alveolar differentiation and maturation. MMP-2 is known to play a crucial role in this process. The aim of this study was to investigate the potential association between levels of MMP2, TIMP-1, TIMP-2 and the development of BPD in preterm infants.

Methods: 27 preterm neonates with a gestational age of $\leq 32$ weeks and birth weight $\leq 1500 \mathrm{~g}$ were included in this prospective study. 14 neonates developed BPD according to $\mathrm{NIH}$ criteria. TAF aspirates were collected on day 1-2 and 5 after birth. Plasma concentrations were measured in umbilical cord blood samples. MMP-2, TIMP-1 and TIMP-2 concentrations were assayed by ELISA.

Results: In the group of neonates with BPD or death initial levels of examined proteins were significantly higher compared to non-BPD preterms. Additionally, in preterm infants TAF levels of MMP2, TIMP-1 and TIMP-2 undergo dynamic changes in the first 5 days of live. Significantly lower levels of MMP-2 and TIMP-2 on day 5 compared to day 1-2 were associated with development of BPD or death. Umbilical cord blood plasma concentrations of MMP-2, TIMP-1 and TIMP-2 did not differ between groups.

Conclusions: Determination of TAF concentrations of MMP-2 and TIMP-2 and observation of their changes in the first days of life in preterm neonates is of prognostic value for development of BPD.

\section{1}

RECOMBINANT HUMAN KGF/PALIFERMIN $®$ AS AN ALTERNATIVE TO GLUCOCORTICOIDSEFFECTS ON PNEUMOCYTE PROLIFERATION AND GENE EXPRESSION IN NEONATAL RATS

W. Bernhard ${ }^{1}$, R. Koslowski ${ }^{2}$, H. Fehrenbach ${ }^{3}$, C.F. Poets ${ }^{1}$

${ }^{1}$ Neonatology, Eberhard-Karls-University, Tübingen, ${ }^{2}$ Physiologic Chemistry, Univ. Dresden, Dresden, ${ }^{3}$ Dept. of Experimental Pneumology, Laboratory of Applied Molecular Biology and Immunologyeibniz Center for Medicine and Biosciences, Borstel, Germany

Background: Surfactant decreases surface tension of pulmonary air:liquid interfaces. It mainly comprizes characteristic phosphatidylcholine (PC) species and proteins SP-A to -D. Betamethasone used to accelerate "lung maturation" is catabolic, while keratinocyte growth factor (KGF), expressed by fibroblasts, is non-catabolic, acts specifically on type-II-pneumocytes and correlates inversely with bronchopulmonary dysplasia incidence.

Aims: To explore the potential of recombinant human KGF (rhKGF, Palifermin $®$ ) on neonatal lung development and surfactant metabolism. To contrast rhKGF with betamethasone effects in vivo.

Methods: Postnatal rats $(\mathrm{d} 1, \mathrm{~d} 5, \mathrm{~d} 19)$ were injected with $\mathrm{rhKGF}(2 \times 5 \mathrm{mg} / \mathrm{kg})$, betamethasone $(2 \times 1 \mathrm{mg} / \mathrm{kg})$ or rhKGF+betamethasone over 48h. Pneumocyte 